\title{
ON 4-MANIFOLDS WITH UNIVERSAL COVERING SPACE A COMPACT GEOMETRIC MANIFOLD
}

\author{
JONATHAN A. HILLMAN
}

(Received 2 June 1992)

Communicated by J. H. Rubinstein

\begin{abstract}
There are 11 closed 4-manifolds which admit geometries of compact type $\left(S^{4}, C P^{2}\right.$ or $\left.S^{2} \times S^{2}\right)$ and two other closely related bundle spaces $\left(S^{2} \tilde{\times} S^{2}\right.$ and the total space of the nontrivial $R P^{2}$-bundle over $S^{2}$ ). We show that the homotopy type of such a manifold is determined up to an ambiguity of order at most 4 by its quadratic 2-type, and this in turn is (in most cases) determined by the Euler characteristic, fundamental group and Stiefel-Whitney classes. In (at least) seven of the 13 cases, a PL 4-manifold with the same invariants as a geometric manifold or bundle space must be homeomorphic to it.
\end{abstract}

1991 Mathematics subject classification (Amer. Math. Soc.): $57 \mathrm{~N} 13$.

Keywords and phrases: 4-manifold, geometry, $k$-invariant, quadratic 2-type, Stiefel-Whitney class.

\section{Introduction}

In this paper we shall attempt to characterize up to homotopy equivalence or homeomorphism the closed 4-manifolds which admit geometries of compact type (and two other closely related bundle spaces), in terms of the Euler characteristic, fundamental group and Stiefel-Whitney classes. The corresponding question for the geometries of noncompact type was considered in two earlier papers [9] and [10]. The solvable aspherical cases correspond to the 4-dimensional infrasolvmanifolds, and these may be characterized up to homeomorphism by

(C) 1993 Australian Mathematical Society $0263-6115 / 93 \$ A 2.00+0.00$ 
the conditions $\chi=0$ and $\pi_{1}$ has a nilpotent normal subgroup of Hirsch length at least 3 [9]. In the other aspherical cases the possible fundamental groups are not yet well understood, but all contain nonabelian free subgroups, and the 5 -dimensional $s$-cobordism theorem is not known over such groups. The three geometries with models homeomorphic to $S^{2} \times R^{2}$ or $S^{3} \times R$ were studied in greater detail in [10].

There are three geometries with compact models, namely $S^{4}, C P^{2}$ and $S^{2} \times S^{2}$. The first two of these are easily dealt with, as there is only one other geometric manifold, namely $R P^{4}$, and for each of the two projective spaces there is one other (non-smoothable) manifold of the same homotopy type. With the geometry $S^{2} \times S^{2}$ we shall consider also the bundle space $S^{2} \tilde{\times} S^{2}$. There are eight $S^{2} \times S^{2}$ manifolds, seven of which are total spaces of bundles with base and fibre each $S^{2}$ or $R P^{2}$, and there are two other such bundle spaces covered by $S^{2} \tilde{x} S^{2}$.

The universal covering space $\tilde{M}$ of a closed 4-manifold $M$ is homeomorphic to $S^{2} \times S^{2}$ if and only if $\pi=\pi_{1}(M)$ is finite, $\chi(M)|\pi|=4$ and $w_{2}(\tilde{M})=0$. (The condition $w_{2}(\tilde{M})=0$ may be restated entirely in terms of $M$, but at somewhat greater length). If these conditions hold and $\pi$ is cyclic then $M$ is homotopy equivalent to an $S^{2} \times S^{2}$-manifold, except perhaps when $\pi=\mathbf{Z} / 2 \mathbf{Z}$ and $M$ is nonorientable, in which case there are at most two other homotopy types. However we have not been able to characterize completely the possible $k$ invariants when $\pi \cong(Z / 2 Z)^{2}$. Moreover, there is a further ambiguity of order at most 4 in determining the homotopy type. If $\chi(M)|\pi|=4$ and $w_{2}(\tilde{M}) \neq 0$ then $\pi=1$ or $M$ is nonorientable and $\pi=\mathbf{Z} / 2 \mathbf{Z}$; in the latter case $\tilde{M} \cong S^{2} \tilde{\times} S^{2}$, there are at most two possible actions of $\pi$ on $\mathbf{Z}^{2}$, for each of which the $k$-invariant lies in a trivial group, and there is a further ambiguity of order at most 2.

The number of homeomorphism classes within each homotopy type is at most two if $\pi=\mathbf{Z} / 2 \mathbf{Z}$ and $w_{2}(\tilde{M})=0$, at most four if $\pi=\mathbf{Z} / 2 \mathbf{Z}$ and $w_{2}(\tilde{M}) \neq 0$ or $\pi \cong \mathbf{Z} / 4 \mathbf{Z}$, and at most eight if $\pi \cong(\mathbf{Z} / 2 \mathbf{Z})^{2}$. We do not know whether there are enough exotic self homotopy equivalences to account for all the normal invariants with trivial surgery obstruction. However a PL 4-manifold with the same homotopy type as a geometric manifold or $S^{2} \tilde{\times} S^{2}$ is homeomorphic to it, in (at least) nine of the 13 cases. (In seven of these cases the homotopy type is determined by the Euler characteristic, fundamental group and Stiefel-Whitney classes). 


\section{The geometries $S^{4}$ and $C P^{2}$}

The unique element of $O(5)=\operatorname{Isom}\left(S^{4}\right)$ of order 2 which acts freely on $S^{4}$ is $-I$. Therefore $S^{4}$ and $R P^{4}$ are the only $S^{4}$-manifolds. The manifold $S^{4}$ is determined up to homeomorphism by the conditions $\chi\left(S^{4}\right)=2$ and $\pi_{1}\left(S^{4}\right)=1$ [4].

The manifold $R P^{4}$ is determined up to homotopy equivalence by the conditions $\chi\left(R P^{4}\right)=1$ and $\pi_{1}\left(R P^{4}\right)=\mathbf{Z} / 2 \mathbf{Z}$ [13]. It follows easily from Theorems 13.A.1 and 13.B.5 of [16] that the surgery obstruction map $c:\left[R P^{4}, G /\right.$ TOP $] \rightarrow$ $\mathrm{Z} / 2 \mathrm{Z}$ is onto and that the structure set $S_{\text {TOP }}\left(R P^{4}\right)$ has two elements. (See the discussion of nonorientable manifolds with fundamental group $Z / 2 Z$ in Section 6 below for more details). As every self homotopy equivalence of $R P^{4}$ is homotopic to the identity [13] there is one fake $R P^{4}$. The fake $R P^{4}$ is not smoothable [14].

There are no nontrivial fixed point free actions on $C P^{2}$, as any self map of $C P^{2}$ has non-zero Lefshetz number, and so it is the only $C P^{2}$-manifold. It is determined up to homotopy equivalence by the conditions $\chi\left(C P^{2}\right)=3$ and $\pi=1$. In this case the surgery obstruction is determined by signatures and maps $\left[C P^{2}, G /\right.$ TOP] onto $\mathrm{Z}$. The structure set $S_{\text {TOP }}\left(C P^{2}\right)$ again has two elements, and as every self homotopy equivalence of $C P^{2}$ is homotopic to the identity there is one fake $C P^{2}$. The fake $C P^{2}$ is also known as the Chern manifold $\mathrm{Ch}$ or $* C P^{2}$, and is not smoothable [4].

\section{The geometry $S^{2} \times S^{2}$}

The manifold $S^{2} \times S^{2}$ is determined up to homeomorphism by the conditions $\chi\left(S^{2} \times S^{2}\right)=4, \pi_{1}\left(S^{2} \times S^{2}\right)=1$ and $w_{2}\left(S^{2} \times S^{2}\right)=0$ [4]. Hence if $M$ is an $S^{2} \times S^{2}$-manifold its fundamental group $\pi$ is finite, $\chi(M)|\pi|=4$ and $w_{2}(\tilde{M})=0$.

The isometry group of $S^{2} \times S^{2}$ is the semidirect product $(O(3) \times O(3)) \tilde{\times}(\mathbf{Z} / 2 \mathbf{Z})$, and is generated by the elements $\tau$, where $\tau(x, y)=(y, x)$, and $(A, B)$, where $A, B \in O(3)$. The involution $\tau$ acts on $O(3) \times O(3)$ by $\tau(A, B) \tau=(B, A)$ for $A, B \in O(3)$. In particular, $(\tau(A, B))^{2}=$ id if and only if $A B=I$, and so such an involution fixes $(x, A x)$, for any $x \in S^{2}$. Thus there are no free $\mathbf{Z} / \mathbf{Z Z}$-actions in which the factors are switched. The element $(A, B)$ generates a free $\mathbf{Z} / 2 \mathbf{Z}$-action if and only if $A^{2}=B^{2}=I$ and at least one of $A, B$ acts freely, that is if $A$ or $B=-I$. After conjugation with $\tau$ if necessary we may assume 
that $B=-I$, and so there are four conjugacy classes in $\operatorname{Isom}\left(S^{2} \times S^{2}\right)$ of free $\mathbf{Z} / 2 \mathbf{Z}$-actions. (The conjugacy classes may be distinguished by the multiplicity $(0,1,2$ or 3$)$ of 1 as an eigenvalue of $A)$. In each case the projection onto the second factor gives rise to a fibre bundle projection from the orbit space to $R P^{2}$, with fibre $S^{2}$.

If the involutions $(A, B)$ and $(C, D)$ generate a free $(\mathbf{Z} / 2 \mathbf{Z})^{2}$-action then $(A C, B D)$ is also a free involution. By the above paragraph, one element of each of these ordered pairs must be $-I$. It follows easily that (after conjugation with $\tau$ if necessary), the $(\mathbf{Z} / 2 \mathbf{Z})^{2}$-actions are generated by pairs $(A,-I)$ and $(-I, I)$, where $A^{2}=I$. Since $A$ and $-A$ give rise to the same subgroup, there are two free $(\mathbf{Z} / 2 \mathbf{Z})^{2}$-actions. The orbit spaces are the total spaces of $R P^{2}$-bundles over $R P^{2}$.

If $(\tau(A, B))^{4}=$ id then $(B A, A B)$ is a fixed point free involution and so $B A=A B=-I$. Since $(A, I) \tau\left(A,-A^{-1}\right)(A, I)^{-1}=\tau(I,-I)$ every free $\mathrm{Z} / 4 \mathrm{Z}$-action is conjugate to the one generated by $\tau(I,-I)$. The orbit space does not fibre over a surface. (See below).

In the next section we shall see that these eight geometric manifolds may be distinguished by their fundamental group and Stiefel-Whitney classes.

\section{Bundle spaces}

There are two $S^{2}$-bundles over $S^{2}$, since $\pi_{1}(S O(3))=\mathbf{Z} / 2 \mathbf{Z}$. The total space $S^{2} \tilde{x} S^{2}$ of the nontrivial $S^{2}$-bundle over $S^{2}$ is determined up to homotopy equivalence by the conditions $\chi\left(S^{2} \tilde{x} S^{2}\right)=4, \pi_{1}\left(S^{2} \tilde{x} S^{2}\right)=1, w_{2}\left(S^{2} \tilde{x} S^{2}\right) \neq 0$ and $\sigma\left(S^{2} \tilde{x} S^{2}\right)=0$. However there is one fake $S^{2} \tilde{x} S^{2}$. The bundle space is homeomorphic to the connected sum $C P^{2} \sharp-C P^{2}$, while the fake version is homeomorphic to $\mathrm{Ch} \sharp-C P^{2}$ and is not smoothable [4].

Since the Kirby-Siebenmann obstruction of a closed 4-manifold is natural with respect to covering maps and dies on passage to 2 -fold coverings, the nonsmoothable manifold $\mathrm{Ch} \sharp-C P^{2}$ admits no nontrivial free involution, and so does not properly cover any other 4-manifold. The following lemma implies that $S^{2} \tilde{x} S^{2}$ admits no orientation preserving free involution, and hence no free action of $\mathbf{Z} / 4 \mathbf{Z}$ or $(\mathbf{Z} / 2 \mathbf{Z})^{2}$.

LEMMA 1. Let $M$ be a closed 4-manifold with fundamental group $\pi=\mathbf{Z} / 2 \mathbf{Z}$ and universal covering space $\tilde{M}$. Then

$$
w_{2}(\tilde{M})=0 \text { if and only if } w_{2}(M)=u^{2} \text { for some } u \in H^{1}(M ; \mathbf{Z} / 2 \mathbf{Z}) ; \text { and }
$$


(ii) if $M$ is orientable and $\chi(M)=2$ then $w_{2}(\tilde{M})=0$ and so $\tilde{M} \cong S^{2} \times S^{2}$.

PROOF. The Cartan-Leray cohomology spectral sequence (with coefficients $\mathbf{Z} / 2 \mathbf{Z}$ ) for the projection $p: \tilde{M} \rightarrow M$ gives an exact sequence

$$
0 \rightarrow H^{2}(\pi ; \mathbf{Z} / 2 \mathbf{Z}) \rightarrow H^{2}(M ; \mathbf{Z} / 2 \mathbf{Z}) \rightarrow H^{2}(\tilde{M} ; \mathbf{Z} / 2 \mathbf{Z})
$$

in which the right hand map is induced by $p$ and has image in the subgroup fixed under the action of $\pi$. Hence $w_{2}(\tilde{M})=p^{*} w_{2}(M)$ is 0 if and only if $w_{2}(M)$ is in the image of $H^{2}(\pi ; \mathbf{Z} / 2 \mathbf{Z})$. Since $\pi=\mathbf{Z} / 2 \mathbf{Z}$ this is so if and only if $w_{2}(M)=u^{2}$ for some $u \in H^{1}(M ; \mathbf{Z} / 2 Z)$.

Suppose now that $M$ is orientable and $\chi(M)=2$. Then $H^{2}(\pi ; \mathbf{Z})=$ $H^{2}(M ; \mathbf{Z})=\mathbf{Z} / 2 \mathbf{Z}$. The integral analogue of the above exact sequence implies that the natural map from $H^{2}(\pi ; \mathbf{Z})$ to $H^{2}(M ; \mathbf{Z})$ is an isomorphism and so $p^{*}\left(H^{2}(\tilde{M} ; \mathbf{Z})\right)=0$. Since $M$ is an orientable 4-manifold, $w_{2}(M)$ is the reduction of an integral class $W_{2}$, by Lemma 6 of [3]. Hence $w_{2}(\tilde{M})$ is the reduction of $p^{*} W_{2}$ and so is 0 . Therefore $\tilde{M} \cong S^{2} \times S^{2}$.

Since $R P^{2}=\mathrm{Mb} \cup D$ is the union of a Möbius band $\mathrm{Mb}$ and a disc $D$, an $F$ bundle over $R P^{2}$ is determined by a bundle over $\mathrm{Mb}$ which restricts to a trivial bundle over $\partial \mathrm{Mb}$, that is by a conjugacy class of elements of order dividing 2 in $\pi_{0}(\operatorname{Homeo}(F))$, together with the class of a gluing map over $\partial \mathrm{Mb}=\partial D$ modulo those which extend across $D$ or $\mathrm{Mb}$, that is an element of a quotient of $\pi_{1}($ Homeo $(F))$. Hence there are four $S^{2}$-bundles over $R P^{2}$.

The orbit space $M=\left(S^{2} \times S^{2}\right) /(A,-I)$ is orientable if and only if $\operatorname{det}(A)=$ -1 . If $A$ has a fixed point $P \in S^{2}$ then the image of $\{P\} \times S^{2}$ in $M$ is an embedded projective plane which represents a non-zero class in $H_{2}(M ; \mathbf{Z} / 2 \mathbf{Z})$. If $A=I$ or is a reflection across a plane, the fixed point set has dimension $>0$ and so this projective plane has self intersection 0 . As the fibre $S^{2}$ intersects this projective plane in one point and has self intersection 0 it follows that the second Wu class $v_{2}(M)=w_{2}(M)+w_{1}(M)^{2}$ is 0 and so $w_{2}(M)=w_{1}(M)^{2}$ in these two cases. If $A$ is a rotation about an axis then the projective plane has self intersection 1. Finally, if $A=-I$ then the image of the diagonal $\left\{(x, x) \mid x \in S^{2}\right\}$ is a projective plane in $M$ with self intersection 1 [5]. Thus in these two cases $v_{2}(M) \neq 0$. Therefore, by part (i) of the lemma, $w_{2}(M)$ is the square of the non-zero element of $H^{1}(M ; \mathbf{Z} / 2 Z)$ if $A=-I$ and is 0 if $A$ is a rotation. Thus these bundle spaces may be distinguished by their Stiefel-Whitney classes, and every $S^{2}$-bundle over $R P^{2}$ is geometric. 
The group $E\left(R P^{2}\right)$ of self homotopy equivalences of $R P^{2}$ is connected and the natural map from $S O(3)$ to $E\left(R P^{2}\right)$ induces an isomorphism on $\pi_{1}$, (cf. [8]). Hence there are two $R P^{2}$-bundles over $S^{2}$, up to fibre homotopy equivalence. The total space of the nontrivial $R P^{2}$-bundle over $S^{2}$ is the quotient of $S^{2} \tilde{x} S^{2}$ by the bundle involution which is the antipodal map on each fibre.

There are two $R P^{2}$-bundles over $R P^{2}$. (The total spaces of each of the latter bundles have fundamental group $(\mathbf{Z} / 2 \mathbf{Z})^{2}$, since $w_{1}: \pi \rightarrow \mathbf{Z} / 2 \mathbf{Z}=$ $\pi_{1}\left(R P^{2}\right)$ restricts nontrivially to the fibre, and so is a splitting homomorphism for the homomorphism induced by the inclusion of the fibre). They may be distinguished by their orientation double covers, and each is geometric.

\section{The action of $\pi$ on $\pi_{2}$}

The two inclusions of $S^{2}$ as factors of $S^{2} \times S^{2}$ determine the standard basis for $\pi_{2}\left(S^{2} \times S^{2}\right)$. Let

$$
J=\left(\begin{array}{ll}
0 & 1 \\
1 & 0
\end{array}\right)
$$

be the matrix of the intersection form $\bullet$ on $\pi_{2}\left(S^{2} \times S^{2}\right)$, with respect to this basis. The group Aut $( \pm \bullet)$ of automorphisms of $\pi_{2}\left(S^{2} \times S^{2}\right)$ which preserve this intersection form up to sign is the dihedral group of order eight, and is generated by the diagonal matrices and $J$ or

$$
K=\left(\begin{array}{cc}
0 & 1 \\
-1 & 0
\end{array}\right)
$$

The subgroup of strict isometries has order four, and is generated by $-I$ and $J$. (Note that the isometry $J$ is induced by the involution $\tau$ ).

Let $f$ be a self-homeomorphism of $S^{2} \times S^{2}$ and let $f_{*}$ be the induced automorphism of $\pi_{2}\left(S^{2} \times S^{2}\right)$. The Lefshetz number of $f$ is $2+\operatorname{trace}\left(f_{*}\right)$ if $f$ is orientation preserving and $\operatorname{trace}\left(f_{*}\right)$ if $f$ is orientation reversing. As any self homotopy equivalence which induces the identity on $\pi_{2}$ has non-zero Lefshetz number, the natural representation of a group $\pi$ of fixed point free self homeomorphisms of $S^{2} \times S^{2}$ into Aut $( \pm \bullet)$ is faithful.

Suppose first that $f$ is a free involution, so $f_{*}^{2}=l$. If $f$ is orientation preserving then $\operatorname{trace}\left(f_{*}\right)=-2$, so $f_{*}=-I$. If $f$ is orientation reversing, then $\operatorname{trace}\left(f_{*}\right)=0$, so

$$
f_{*}= \pm J K= \pm\left(\begin{array}{cc}
1 & 0 \\
0 & -1
\end{array}\right)
$$


Note that if $f^{\prime}=\tau f \tau$ then $f_{*}^{\prime}=-f_{*}$, so after conjugation by $\tau$ if necessary we may assume that $f_{*}=J K$.

If $f$ generates a free $\mathbf{Z} / 4 \mathrm{Z}$-action the induced automorphism must be $\pm K$. Note that if $f^{\prime}=\tau f \tau$ then $f_{*}^{\prime}=-f_{*}$, so after conjugation by $\tau$ if necessary we may assume that $f_{*}=K$.

Since the orbit space of a fixed point free action of $(\mathbf{Z} / 2 \mathbf{Z})^{2}$ on $S^{2} \times S^{2}$ has Euler characteristic 1, it is nonorientable, and so the action is generated by two commuting involutions, one of which is orientation preserving and one of which is not. Since the orientation preserving involution must act via $-I$ and the orientation reversing involutions must act via $\pm J K$ the action of $(Z / 2 Z)^{2}$ is essentially unique.

The standard inclusions of $S^{2}=C P^{1}$ into the summands of

$$
C P^{2} \sharp-C P^{2} \cong S^{2} \tilde{x} S^{2}
$$

determine a basis for $\pi_{2}\left(S^{2} \tilde{x} S^{2}\right) \cong \mathbf{Z}^{2}$. Let $\tilde{J}=\left(\begin{array}{cc}1 & 0 \\ 0 & -1\end{array}\right)$ be the matrix of the intersection form $\tilde{\bullet}$ on $\pi_{2}\left(S^{2} \tilde{x} S^{2}\right)$ with respect to this basis. The group $\operatorname{Aut}( \pm \tilde{\bullet})$ of automorphisms of $\pi_{2}\left(S^{2} \tilde{x} S^{2}\right)$ which preserve this intersection form up to sign is the dihedral group of order eight, and is also generated by the diagonal matrices and $J=\left(\begin{array}{ll}0 & 1 \\ 1 & 0\end{array}\right)$. The subgroup of strict isometries has order four, and consists of the diagonal matrices. A nontrivial group of fixed point free self homeomorphisms of $S^{2} \tilde{x} S^{2}$ must have order 2 , since $S^{2} \tilde{\times} S^{2}$ admits no fixed point free orientation preserving involution. If $f$ is an orientation reversing free involution of $S^{2} \tilde{x} S^{2}$ then $f_{*}= \pm J$. The automorphism induced by the fibrewise antipodal map is $-J$, as this map clearly changes the sign of the homotopy class of the fibre, which has self intersection 0 . Is $J$ also realizable by an orientation reversing involution?

\section{The homotopy type}

We shall assume henceforth that $M$ is a closed connected 4-manifold with finite fundamental group $\pi$ and that $\chi(M)|\pi|=4$. Moreover we may choose a basis for $\pi_{2}(M) \cong \mathbf{Z}^{2}$ so that the corresponding basis of the intersection form on $\pi_{2}(M)$ is $J$ or $\tilde{J}$, as in Section 4. The quadratic 2-type of $M$ is the quadruple $\left[\pi, \pi_{2}(M), \kappa(M), S(\tilde{M})\right]$, where $\kappa(M) \in H^{3}\left(\pi ; \pi_{2}(M)\right)$ is the first $k$-invariant of $M$ and $S(\tilde{M})$ is the intersection form on $\pi_{2}(M)=H_{2}(\tilde{M} ; \mathbf{Z})$. Two such quadruples $[\pi, \Pi, \kappa, S]$ and $\left[\pi^{\prime}, \Pi^{\prime}, \kappa^{\prime}, S^{\prime}\right]$ are equivalent if there is 
an isomorphism $\alpha: \pi \rightarrow \pi^{\prime}$ and an isometry $\beta:(\Pi, S) \rightarrow\left(\Pi^{\prime}, S^{\prime}\right)$ which is $\alpha$-equivariant (that is, such that $\beta(g m)=\alpha(g) \beta(m)$ for all $g \in \pi$ and $m \in \Pi$ ) and $\beta_{*} \kappa=\alpha^{*} \kappa^{\prime}$ in $H^{3}\left(\pi, \alpha^{*} \Pi^{\prime}\right)$. If $M$ is orientable and $\pi$ is cyclic then the equivalence class of the quadratic 2-type determines the homotopy type [6]. In particular, if $\pi=1$ the manifold $M$ is homotopy equivalent to $S^{2} \times S^{2}, S^{2} \tilde{\times} S^{2}$ or $C P^{2} \sharp C P^{2}$. (See Addendum.)

The argument of [6] has been reformulated and extended in Theorem II.7.12 of [1]. If $M$ is non-orientable we may adapt Baues' argument by using the orientation $\pi$-module $\tilde{\mathbf{Z}}$ and the $w_{1}$-twisted transfer $\tilde{\mathrm{tr}}: \Gamma\left(\pi_{2}\right) \otimes_{\pi} \tilde{\mathbf{Z}} \rightarrow \Gamma\left(\pi_{2}\right)$, defined by $\operatorname{tr}(x \otimes 1)=\Sigma_{g \in \pi} w_{1}(g) x \cdot g$, instead of the augmentation module $\mathbf{Z}$ and the ordinary transfer. In all cases $\Gamma\left(\mathbf{Z}^{2}\right) \cong \Gamma(\mathbf{Z}) \oplus \Gamma(\mathbf{Z}) \oplus(\mathbf{Z} \odot \mathbf{Z}) \cong \mathbf{Z}^{3}$, as an abelian group.

LEMMA 2. Let $M$ be a closed 4-manifold with fundamental group $\pi=\mathbf{Z} / 2 \mathbf{Z}$ and universal covering space $S^{2} \times S^{2}$. Then the first $k$-invariant of $M$ is a non-zero element of $H^{3}\left(\pi ; \pi_{2}(M)\right)$.

ProOf. Let $P_{2}(M)$ be the second stage of the Postnikov tower for $M$, and let $c_{M}=g_{M} f_{M}$ be the factorization of the classifying map $c_{M}: M \rightarrow K(\pi, 1)=$ $R P^{\infty}$ through $f_{M}: M \rightarrow P_{2}(M)$ and $g_{M}: P_{2}(M) \rightarrow K(\pi, 1)$. The first $k$-invariant is the primary obstruction to the existence of a cross-section to $c_{M}$ and is the only obstruction to the existence of such a cross-section for $g_{M}$.

The only non-zero differentials in the Cartan-Leray cohomology spectral sequence (with coefficients $\mathbf{Z} / 2 \mathbf{Z}$ ) for the projection $p: \tilde{M} \rightarrow M$ are at the $E_{3}^{* *}$ level. By the results of Section $4, \pi$ acts trivially on $H^{2}(\tilde{M} ; \mathbf{Z} / 2 Z)$, since $\tilde{M}=S^{2} \times S^{2}$. Therefore $E_{3}^{22}=E_{2}^{22} \cong(\mathbf{Z} / 2 \mathbf{Z})^{2}$ and $E_{3}^{50}=E_{2}^{50}=\mathbf{Z} / 2 \mathbf{Z}$. Hence $E_{\infty}^{22} \neq 0$, so $E_{\infty}^{22}$ maps onto $H^{4}(M ; \mathbf{Z} / 2 \mathbf{Z})=\mathbf{Z} / 2 \mathbf{Z}$ and

$$
d_{3}^{12}: H^{1}\left(\pi ; H^{2}(\tilde{M} ; \mathbf{Z} / 2 \mathbf{Z})\right) \rightarrow H^{4}(\pi ; \mathbf{Z} / 2 \mathbf{Z})
$$

must be onto. But in this region the spectral sequence is identical with the corresponding spectral sequence for $P_{2}(M)$. It follows that the image of $H^{4}(\pi ; \mathbf{Z} / 2 \mathbf{Z})=\mathbf{Z} / 2 \mathbf{Z}$ in $H^{4}\left(P_{2}(M) ; \mathbf{Z} / 2 \mathbf{Z}\right)$ is 0 , and so $g_{M}$ does not admit a cross-section. Thus $\kappa(M) \neq 0$.

If $\pi=\mathbf{Z} / 2 Z$ and $M$ is orientable then $\pi$ acts via $-I$ on $\mathbf{Z}^{2}$ and the $k$-invariant is a non-zero element of $H^{3}\left(\mathbf{Z} / 2 \mathbf{Z} ; \pi_{2}(M)\right)=(\mathbf{Z} / 2 \mathbf{Z})^{2}$. The isometry which transposes the standard generators of $\mathbf{Z}^{2}$ is $\pi$-linear, and so there are just two equivalence classes of quadratic 2 -types to consider. The $k$-invariant which is 
invariant under transposition is realised by $\left(S^{2} \times S^{2}\right) /(-I,-I)$, while the other $k$-invariant is realized by the orientable bundle space with $w_{2}=0$ [6]. Thus $M$ must be homotopy equivalent to one of these spaces.

If $\pi=\mathbf{Z} / 2 \mathbf{Z}, M$ is nonorientable and $w_{2}(\tilde{M})=0$ then $H^{3}\left(\pi ; \pi_{2}(M)\right)=$ $\mathbf{Z} / 2 \mathbf{Z}$ and there is only one quadratic 2-type to consider. The product space $S^{2} \times R P^{2}$ is characterized by the additional conditions that $w_{2}(M)=w_{1}(M)^{2}$ and that there is an element $u \in H^{2}(M ; Z)$ which generates an infinite cyclic direct summand and is such that $u \cup u=0$ [8]. The nontrivial nonorientable $S^{2}$-bundle over $R P^{2}$ has $w_{2}(M)=0$. As ker $\tilde{\mathrm{tr}} \cong(\mathbf{Z} / 2 \mathbf{Z})^{2}$ there are at most two other homotopy types of 4-dimensional Poincaré complexes within this quadratic 2-type. (There are in fact four such homotopy types, one of which is not realizable by a closed 4 -manifold [11]).

If $\pi=\mathbf{Z} / 2 \mathbf{Z}$ and $w_{2}(\tilde{M}) \neq 0$ then there are two possible actions of $\pi$ on $\mathbf{Z}^{2}$; we do not know whether both can be realized, but in either case, $H^{3}\left(\pi_{1} ; \pi_{2}(M)\right)=0$. (Note that in this case the argument of Lemma 2 breaks down because $E_{\infty}^{22}=0$ ). The nontrivial $R P^{2}$-bundle over $S^{2}$ is characterized by the additional condition that there is an element $u \in H^{2}(M ; Z)$ which generates an infinite cyclic direct summand and such that $u \cup u=0$ [8]. As ker $\tilde{\mathrm{r}}=\mathbf{Z} / 2 \mathbf{Z}$ there is at most one other homotopy type with the same quadratic 2-type as this bundle space.

If $\pi \cong \mathbf{Z} / 4 \mathbf{Z}$ then $H^{3}\left(\pi ; \pi_{2}(M)\right) \cong \operatorname{ker}\left(I+f_{*}+f_{*}^{2}+f_{*}^{3}\right) /\left(I-f_{*}\right)=$ $\mathbf{Z}^{2} /(I-K) \mathbf{Z}^{2}=\mathbf{Z} / 2 \mathbf{Z}$. The $k$-invariant is non-zero, since it restricts to the $k$-invariant of the orientation double cover. In this case $\tilde{\text { tr }}$ is injective and so $M$ is homotopy equivalent to $\left(S^{2} \times S^{2}\right) / \tau(I,-I)$.

Suppose now that $\pi \cong(\mathbf{Z} / 2 \mathbf{Z})^{2}$ is the diagonal subgroup of $\operatorname{Aut}( \pm \bullet)<$ $\mathrm{GL}(2, \mathbf{Z})$, and let $\alpha$ be the automorphism induced by conjugation by $J$. The standard generators of $\pi_{2}(M)=\mathbf{Z}^{2}$ generate complementary $\pi$-submodules, so that $\pi_{2}(M)$ is the direct sum $\tilde{\mathbf{Z}} \oplus \alpha^{*} \tilde{\mathbf{Z}}$ of two infinite cyclic modules. The isometry $\beta=J$ which transposes the factors is $\alpha$-equivariant, and $\pi$ and $V=\{ \pm I\}$ act nontrivially on each summand. If $\rho$ is the kernel of the action of $\pi$ on $\tilde{\mathbf{z}}$ then $\alpha(\rho)$ is the kernel of the action on $\alpha^{*} \tilde{\mathbf{Z}}$, and $\rho \cap \alpha(\rho)=1$. As the projection of $\pi=\rho \oplus V$ onto $V$ is compatible with the action, $H^{*}(V ; \tilde{\mathbf{z}})$ is a direct summand of $H^{*}(\pi ; \tilde{\mathbf{z}})$. This implies in particular that the differentials in the LHS spectral sequence $H^{p}\left(V ; H^{q}(\rho ; \tilde{\mathbf{Z}})\right) \Rightarrow H^{p+q}(\pi ; \tilde{\mathbf{z}})$ which end on the row $q=0$ are all 0 . Hence $H^{3}\left(\pi_{1} ; \tilde{\mathbf{z}}\right) \cong H^{1}(V ; \mathbf{Z} / 2 \mathbf{Z}) \oplus H^{3}(V ; \tilde{\mathbf{Z}}) \cong(\mathbf{Z} / 2 \mathbf{Z})^{2}$. Similarly $H^{3}\left(\pi_{1} ; \alpha^{*} \tilde{\mathbf{Z}}\right) \cong(\mathbf{Z} / 2 \mathbf{Z})^{2}$, and so $H^{3}\left(\pi_{1} ; \pi_{2}(M)\right) \cong(\mathbf{Z} / 2 \mathbf{Z})^{4}$. The $k$-invariant must restrict to the $k$-invariant of each double cover, which must be non-zero, by Lemma 2. As $H^{3}(\rho ; \tilde{\mathbf{z}})=H^{3}\left(\alpha(\rho) ; \alpha^{*} \tilde{\mathbf{Z}}\right)=0$ and $H^{3}\left(\rho ; \alpha^{*} \tilde{\mathbf{Z}}\right)=$ 
$H^{3}(\alpha(\rho) ; \tilde{\mathbf{Z}})=\mathbf{Z} / 2 \mathbf{Z}$, there are at most four possible $k$-invariants. Moreover, the automorphism $\alpha$ and the isometry $\beta=J$ act on the $k$-invariant by transposing the factors. As there are either zero or two such $k$-invariants which are invariant under this transposition, there are either two or three equivalence classes of quadratic 2-types to be considered. In this case $\operatorname{ker} \tilde{\mathrm{tr}} \cong(\mathbf{Z} / 2 \mathbf{Z})^{2}$, and so there are at most 12 homotopy types of such manifolds.

\section{Surgery}

In the present context, every homotopy equivalence is simple since Wh $(\pi)=$ 0 for all groups $\pi$ of order $\leq 4$ [7].

Suppose first that $\pi=\mathbf{Z} / 2 \mathbf{Z}$. Then $H^{1}(M ; \mathbf{Z} / 2 Z)=\mathbf{Z} / 2 Z$ and $\chi(M)=2$, so $H^{2}(M ; \mathbf{Z} / 2 \mathbf{Z}) \cong(\mathbf{Z} / 2 \mathbf{Z})^{2}$. If $M$ is orientable then $[M, G / \mathrm{TOP}] \cong \mathbf{Z} \oplus(\mathbf{Z} / 2 \mathbf{Z})^{2}$. The surgery obstruction groups are $L_{5}(\mathbf{Z} / 2 \mathbf{Z},+)=0$ and $L_{4}(\mathbf{Z} / 2 \mathbf{Z},+) \cong$ $\mathbf{Z}^{2}$, where the surgery obstructions are determined by the signature and the signature of the double cover, by Theorem 13.A.1 of [16]. If $M$ is nonorientable then $[M, G / \mathrm{TOP}] \cong(\mathrm{Z} / 2 \mathrm{Z})^{3}$, and the surgery obstruction of a 4-dimensional normal map $g: M \rightarrow G /$ TOP is the Kervaire-Arf invariant $c(g) \in L_{4}(\mathbf{Z} / 2 \mathbf{Z},-)=\mathbf{Z} / 2 \mathbf{Z}$, while $L_{5}(\mathbf{Z} / 2 \mathbf{Z},-)=0$, by Theorem 13.A.1 of [16]. Now $c(g)=w(M) g^{*} \kappa[M]=\left(w_{2}(M) \cup g^{*} k_{2}+g^{*} S q^{2} k_{2}\right)[M]=$ $\left(\left(w_{2}(M)+v_{2}(M)\right) \cup g^{*} k_{2}\right)[M]=\left(w_{1}(M)^{2} \cup g^{*} k_{2}\right)[M]$, by Theorem 13.B.5 of [16]. As $w_{1}(M)$ is not the reduction of a class in $H^{1}(M ; \mathbf{Z} / 4 \mathbf{Z})$ its square $w_{1}(M)^{2}$ is non-zero; as every element of $H^{2}(M ; \mathbf{Z} / 2 \mathbf{Z})$ is equal to $g^{*} k_{2}$ for some such $g$ the map $c:[M, G / \mathrm{TOP}] \rightarrow Z / 2 Z$ is onto. Hence it follows from the surgery exact sequence that $S_{\text {TOP }}(M) \cong(\mathbf{Z} / 2 \mathbf{Z})^{2}$ whenever $\pi=\mathbf{Z} / 2 \mathbf{Z}$.

The $\mathbf{Z} / 2 \mathbf{Z}$-Hurewicz homomorphism from $\pi_{2}(M)$ to $H_{2}(M ; \mathbf{Z} / 2 \mathbf{Z}) \cong(\mathbf{Z} / 2 \mathbf{Z})^{2}$ has cokernel $H_{2}(\pi ; \mathbf{Z} / 2 \mathbf{Z})=\mathbf{Z} / 2 \mathbf{Z}$. Therefore there is a map $\beta: S^{2} \rightarrow M$ such that $\beta_{*}\left[S^{2}\right] \neq 0$ in $H_{2}(M ; \mathbf{Z} / 2 \mathbf{Z})$. If, moreover, $w_{2}(\tilde{M})=0$ then $\beta^{*} w_{2}(M)=0$, since $\beta$ factors through $\tilde{M}$. Let $f_{\beta}=\left(\operatorname{id}_{M}, \beta \eta S \eta\right) s$ where $\eta$ is the Hopf map and $s: M \rightarrow M \vee S^{4}$ is the pinch map obtained by shrinking the boundary of a 4-disc in $M$. Then $f_{\beta}$ is a self homotopy equivalence of $M$ and its normal invariant in $[M, G / \mathrm{TOP}]$ is nontrivial, by the argument of Theorem 16.5 of [16]. (See also Section 5 of [2]). Hence there are at most two homeomorphism classes within the homotopy type of $M$ if $\pi=\mathbf{Z} / 2 \mathbf{Z}$ and $w_{2}(\tilde{M})=0$.

When $\pi \cong \mathbf{Z} / 4 \mathbf{Z}$ or $(\mathbf{Z} / 2 \mathbf{Z})^{2}$ the manifold $M$ is nonorientable, since $\chi(M)=$ 1. If $\pi \cong \mathbf{Z} / 4 \mathrm{Z}$ then $[M, G / \mathrm{TOP}] \cong(\mathrm{Z} / 2 \mathrm{Z})^{2}$ and the surgery obstruction groups $L_{4}(\mathbf{Z} / 4 \mathbf{Z},-)$ and $L_{5}(\mathbf{Z} / 4 \mathrm{Z},-)$ are both 0 , by Theorem 3.4.5 of [17]. 
Hence $S_{\text {TOP }}(M) \cong(\mathbf{Z} / 2 Z)^{2}$. If $\pi \cong(\mathbf{Z} / 2 \mathbf{Z})^{2}$ then $[M, G / T O P] \cong(Z / 2 Z)^{4}$ and the surgery obstruction groups are $L_{5}\left((Z / 2 Z)^{2},-\right)=0$ and $L_{4}\left((Z / 2 Z)^{2},-\right)=$ $\mathbf{Z} / 2 \mathbf{Z}$, by Theorem 3.5 .1 of [17]. The homomorphism $w_{1}(M):(\mathbf{Z} / 2 \mathbf{Z})^{2} \rightarrow$ $\mathbf{Z} / 2 \mathbf{Z}$ induces an isomorphism from $L_{4}\left((\mathbf{Z} / 2 \mathbf{Z})^{2},-\right)$ onto $L_{4}(\mathbf{Z} / 2 \mathbf{Z},-)$ and so the surgery obstruction is again given by the Kervaire-Arf invariant. We now find that $S_{\text {ToP }}(M) \cong(Z / 2 Z)^{3}$. The argument above for the existence of exotic self homotopy equivalences does not apply as the $\mathbf{Z} / 2 \mathbf{Z}$-Hurewicz homomorphism is 0 in these cases.

The image of $[M, G / \mathrm{PL}]$ in $[M, G / \mathrm{TOP}]$ is a subgroup of index 2 (see Section 15 of [15]). It follows that if $M$ is the total space of an $S^{2}$-bundle over $R P^{2}$ then any homotopy equivalence $f: N \rightarrow M$ where $N$ is also PL is homotopic to a homeomorphism. (For then $S_{\text {TOP }}(M)$ has order 4, and the nontrivial element of the image of $S_{\mathrm{pL}}(M)$ is represented by an exotic self homotopy equivalence of $M$. The case $M=S^{2} \times R P^{2}$ was treated in [12]). This is also true if $M=S^{4}$, $R P^{4}, C P^{2}, S^{2} \times S^{2}$ or $S^{2} \tilde{x} S^{2}$. Is it true for the nontrivial $R P^{2}$-bundle over $S^{2}$ and the remaining three $S^{2} \times S^{2}$-manifolds?

\section{Addendum}

The manifold $C P^{2} \sharp C P^{2}$ and $C P^{2} \sharp C h$ also have $\pi=1, \chi=4$ and $w_{2} \neq 0$. However, it is not hard to see that they do not admit any free involutions.

\section{References}

[1] H. J. Baues, 'Combinatorial homotopy and 4-dimensional complexes', in: De Gruyter Expositions in Mathematics 2 (de Gruyter, Berlin - New York, 1991).

[2] T. D. Cochran and N. Habegger, 'On the homotopy theory of simply-connected fourmanifolds', Topology 29 (1990), 419-440.

[3] D. Frank, 'The signature mod 8', Comment. Math. Helv. 48 (1973), 520-524.

[4] M. H. Freedman and F. R. Quinn, Topology of Four-Manifolds (Princeton University Press, Princeton N.J., 1990).

[5] N. Habegger, 'Une variété de dimension 4 avec forme d'intersection paire et signature -8', Comment. Math. Helv. 57 (1982), 22-24.

[6] I. Hambleton and M. Kreck, 'On the homotopy classification of topological 4-manifolds with finite fundamental group', Math. Ann. 280 (1988), 85-104.

[7] G. Higman, 'The units of group rings', Proc. London Math. Soc. 46 (1940), 231-248.

[8] J. A. Hillman, 'On 4-manifolds homotopy equivalent to surface bundles over surfaces', Top. Appl. 40 (1991), 275-286. 
[9] _..., 'Geometries on 4-manifolds, Euler characteristic and elementary amenable groups', in: Proceedings of the International Conference on Knots, Osaka 1990 (de Gruyter, Berlin, 1992) pp. 25-46.

[10] _ 'On 4-manifolds with universal covering space $S^{2} \times R^{2}$ or $S^{3} \times R$ ', Top. Appl. (to appear).

[11] M. H. Kim, S. Kojima and F. Raymond, 'Homotopy invariants of nonorientable 4manifolds', Trans. Amer. Math. Soc. 333 (1992), 71-83.

[12] T. Matsumoto, 'On homotopy equivalences of $S^{2} \times R P^{2}$ to itself', J. Math. Kyoto University 19 (1979), 1-17.

[13] P. Olum, 'Mappings of manifolds and the notion of degree', Ann. Math. 58 (1953), 458-480.

[14] D. Rubermann, 'Invariant knots of free involutions of $S^{4}$ ', Top. Appl. 18 (1984), 217-224.

[15] L. C. Siebenmann, 'Topological manifolds', in: Proceedings of the International Congress of Mathematicians, Nice, 1970 (Gauthier-Villars, Paris, 1971), vol. 2 pp. 133-163.

[16] C. T. C. Wall, Surgery on Compact Manifolds (Academic Press, London, 1970).

[17] _ 'Classification of hermitian forms: VI group rings', Ann. Math. 103 (1976), 1-80.

School of Mathematics and Statistics

The University of Sydney

Sydney, NSW 2006

AUSTRALIA 